\title{
Économie et politiques éducatives
}

Nécessité et contraintes

Economy and educational policies. Necessity and constraints

Economía y políticas educativas. Necesidad y dificultades

\section{Pierre Laderrière}

\section{OpenEdition \\ Journals}

Édition électronique

URL : http://journals.openedition.org/ries/3005

DOI : 10.4000/ries.3005

ISSN : 2261-4265

\section{Éditeur}

Centre international d'études pédagogiques

\section{Édition imprimée}

Date de publication : 1 juin 1999

Pagination : 153-164

ISSN : 1254-4590

\section{Référence électronique}

Pierre Laderrière, "Économie et politiques éducatives », Revue internationale d'éducation de Sèvres [En ligne], 22 | 1999, mis en ligne le 13 mai 2013, consulté le 10 décembre 2020. URL : http:// journals.openedition.org/ries/3005; DOI : https://doi.org/10.4000/ries.3005

Ce document a été généré automatiquement le 10 décembre 2020.

(c) Tous droits réservés 


\title{
Économie et politiques éducatives
}

\author{
Nécessité et contraintes \\ Economy and educational policies. Necessity and constraints \\ Economía y políticas educativas. Necesidad y dificultades
}

\section{Pierre Laderrière}

1 L'analyse des articles précédents ne semble laisser aucun doute sur l'existence d'une dimension économique de plus en plus prégnante des politiques éducatives et de formation. Afin de rappeler que l'obsolescence rapide des savoirs généraux et des compétences particulières dans un contexte d'évolution sociotechnique accélérée implique de replacer ces politiques dans un cadre d'apprentissage à vie, on a ajouté cidessus aux "politiques éducatives » la notion de "politique de formation ». Dans la suite du texte, on emploiera, pour plus de simplicité, l'expression large de "politique éducative" intégrant cette politique de formation. Ceci signifie que la période d'enseignement ou/et de formation initiale n'est plus que le socle fondateur des divers apprentissages pouvant intervenir au cours de la vie, qu'ils aient un caractère à dominante personnelle ou à dominante professionnelle.

2 L'approche économique des politiques éducatives a toujours présenté des difficultés à cause de la multi-dimensionnalité de ces politiques. Elles concernent, en effet, tant le développement individuel stricto sensu que le développement du citoyen, du parent, du corps, de la sensibilité artistique, du producteur, etc., tous les éléments pouvant avoir un poids variable selon les contextes nationaux. Or, à des degrés divers, chacun des éléments peut donner naissance à des "externalités ", c'est-à-dire des effets de bien-être ou de revenu additionnel qui peuvent profiter à l'ensemble de la collectivité, mais ne sont pas aisément quantifiables. On est, dans ce cas, vite confronté à des limites méthodologiques ou à l'inaptitude des outils statistiques disponibles, comme le souligne l'article de JeanJacques Paul. Cette très grande difficulté de maîtrise des bénéfices individuels et des bénéfices sociaux des politiques éducatives, afin d'en affiner l'évaluation des résultats et de les administrer de la manière la plus adéquate possible, ne doit pas néanmoins masquer qu'il existe depuis longtemps des instruments divers à la disposition des décideurs politico-administratifs pour enraciner la dimension économique dans la 
conduite de ces politiques. Or, pour diverses raisons, cette nécessité n'est pas toujours franchement reconnue ou, si elle l'est, on ne se donne pas toujours les moyens pouvant permettre de vaincre les obstacles s'opposant à l'utilisation de l'approche économique des politiques éducatives. Ce sont cette nécessité et les contraintes qui lui sont liées qui seront examinées ci-après.

\section{L'enracinement de la dimension économique}

3 Il peut sembler paradoxal d'être obligé de régulièrement justifier la nécessité d'une approche économique des politiques éducatives correspondant à la dimension économique même de ces politiques et des systèmes les mettant en œuvre. Que recouvre, très grossièrement, cette dimension qui, par exemple, est depuis leur origine reconnue au niveau international par des organismes opérationnels, comme la Banque mondiale, ou des organismes de réflexion, comme l'Organisation de coopération et de développement économique (OCDE) ? En premier lieu, cette dimension concerne l'une des finalités des systèmes éducatifs : la formation des "producteurs ", ce terme générique correspondant aux qualifications et compétences offertes à des individus appelés à intégrer la population active et à s'y maintenir ou à s'y réintégrer. En second lieu, cette dimension intéresse la gestion optimale des divers ressources allouées tant par la collectivité que les individus pour le fonctionnement d'ensemble des systèmes éducatifs et le suivi des politiques mises en œuvre à cet effet.

\section{Le premier volet : les compétences}

4 Les divers contextes historiques et culturels de naissance et d'évolution des systèmes éducatifs, soit n'ont pas toujours mis en avant l'importance de l'acquisition de qualifications et de compétences spécifiques pour s'insérer dans la vie active, soit, tout en leur reconnaissant une place plus ou moins importante, ont parfois tendu à l'isoler de l'ensemble du système.

\section{Rappel historique}

5 La relative "ghettoïsation » de ce qui a été qualifié de formation professionnelle des adultes, a plusieurs origines : l'organisation et le lieu principal de formation (comme dans le système dual des pays germanophones), l'orientation vers les premiers niveaux de ce type d'enseignement de groupes socioculturels en échec scolaire (pays de culture latine), l'accès aux niveaux supérieurs de cet enseignement de groupes socioculturels favorisés, à même d'en financer tout ou partie du coût (écoles supérieures de commerce ou de gestion, voire certaines écoles d'ingénieurs prestigieuses des pays occidentaux), l'obsolescence de plus en plus rapide de certains savoir-faire opposés à la permanence des savoirs de base, etc. Mais ce qui a beaucoup prévalu, après la Seconde Guerre mondiale dans de nombreux pays, ce fut une réticence à franchement reconnaitre, d'une part, la multi-dimensionnalité des systèmes et, d'autre part, la place importante à donner à leur production de «qualifications » et « compétences ». Pour les défenseurs d'une définition étroite de la culture individuelle et de son développement, c'était porter atteinte à cette culture que de l'aborder conjointement avec la formation professionnelle, voire traiter comme un tout les notions parfois trop « rabâchées » de «savoir, savoir-faire et savoir- 
être ». Il faut reconnaître que le manque de souplesse des politiques axées sur une articulation étroite entre l'offre et la demande de main-d'œuvre qualifiée ont plus ou moins entraîné leur échec et remis en question une approche trop simpliste de cette articulation. Cet échec n'a fait que renforcer la critique tant d'un certain nombre de spécialistes que de la majorité des enseignants des disciplines générales, contre des « dérives utilitaristes » des systèmes éducatifs. Mais, dans le même temps, on découvrait à l'occasion de certains sondages auprès des parents d'élèves que les parcours scolaires étaient très souvent considérés comme le moyen d'accéder ultérieurement à un emploi, élément-clé d'intégration sociale. Les Trente glorieuses ont ajouté à la confusion en favorisant - au moins dans les pays les plus industrialisés - un développement encore jamais atteint des systèmes éducatifs, les autres groupes de pays bénéficiant eux-mêmes d'une certaine expansion en ce domaine, comme le montre l'article de François Orivel. Ce développement a eu néanmoins son revers en masquant certains déséquilibres - parfois très important - entre l'offre et la demande de qualifications. Et, comme à l'accoutumée, c'est la crise d'ajustement structurel, qu'ont connue de nombreux pays dès la moitié des années soixante-dix, qui a joué le rôle de révélateur. L'analyse du développement du chômage a souvent montré que des diplômes de niveaux élevés pouvaient en protéger certains groupes (avec des nuances soulignées dans l'article sur la Suisse par Edo Poglia) tout comme l'optimisation de la relation théorie-pratique dans la formation professionnelle (voir, de nouveau, les différences régionales suisses de chômage des jeunes selon l'importance du "système dual », dans l'article de Siegfried Hanhart). Dans le même temps, coexistait dans certains pays un chômage très élevé et des déficits sectoriels de qualifications révélant, au-delà même de la question toujours débattue de l'éventuelle raréfaction du travail dans les sociétés avancées, une difficulté persistante d'ajustement du nombre et de la qualité des diplômés aux besoins nécessairement changeants de la société.

\section{L'évolution récente}

6 C'est précisément l'évolution de ces besoins qui semble bouleverser plus profondément cette relation et qui est de plus en plus reconnue ouvertement par les diverses parties prenantes. En premier lieu, il y a une plus grande reconnaissance que la culture générale initialement acquise dans le système scolaire est indispensable à une réelle intégration sociale, en particulier si cette dernière doit se faire par l'accès à un emploi. Il pourra s'agir à la fois d'une culture dite « de base » (lire, écrire, compter, premiers fondements d'un raisonnement analytique, etc.) et d'une culture de plus haut niveau fondée à la fois sur des capacités d'abstraction, de maitrise de la relation théorie-pratique, etc. et sur l'acquisition de langages particuliers de communication, comme les langues étrangères ou les nouvelles technologies de l'information. En second lieu, la plus grande complexité de la vie en société et la nécessité d'en résoudre les problèmes, mais également de les faire émerger, impliquent une plus grande interdisciplinarité des programmes d'enseignement et de formation et la démonstration de leur assimilation dans des projets pédagogiques cohérents. En troisième lieu, plus qu'autrefois, les diverses catégories d'employeurs requièrent ce que l'on a qualifié de « compétences transversales », comme les capacités de s'intégrer à un groupe et de travailler en équipe, de faire montre de ponctualité ou de suivi dans les tâches à accomplir, etc. En quatrième lieu, dans un contexte où les divers types d'employeurs sont confrontés soit à une compétition mondiale soit à la nécessité de réduire les coûts du bien ou service offert, tout en 
maintenant un haut niveau de qualité, on tend, au total, à réclamer des diplômés non seulement un niveau déterminé de qualification, mais également des compétences particulières garantissant leur employabilité, y compris à long terme, grâce à une formation professionnelle continue assise tant sur une formation de base « ouverte » que sur l'expérience professionnelle acquise et validée. Cette évolution montre, en définitif, qu'il est vain d'opposer le quantitatif au qualitatif et que, dans ce domaine, nous pourrions avancer que le qualitatif prime, même si enquêtes et analyses utilisant initialement des méthodes quantitatives se révèlent nécessaires, comme le montrent bien les études récentes de l'OCDE sur le niveau de littéracie des adultes, citées dans la bibliographie annexe à ce dossier. En outre, cette évolution postule, ainsi que le démontre l'article de Martine Möbus et Éric Verdier comparant les procédures allemandes et françaises, un dialogue constant des différents acteurs et partenaires impliqués dans la mise en œuvre et le suivi des programmes d'enseignement, dialogue reposant nécessairement sur une base de données et des analyses constamment mises à jour.

\section{Le second volet : la combinaison optimale des ressources}

7 Parallèlement à l'évolution évoquée ci-dessus concernant les relations nouvelles entre formation et emploi et qui s'est étendue sur une trentaine d'années, on a constaté une approche de plus en plus approfondie du problème des ressources à affecter au fonctionnement des systèmes éducatifs et à leur utilisation la plus rationnelle possible. En premier lieu, l'allocation de moyens financiers à tel ou tel niveau ou tel ou tel type d'enseignement a fait l'objet d'un traitement plus sophistiqué du point de vue méthodologique, à partir, en particulier, d'analyses de taux de rendement et de coûtbénéfice. Ces dernières prennent naturellement en compte certains éléments mentionnés ci-dessus au titre des compétences à acquérir et c'est sans surprise, par exemple, que les spécialistes voient confirmer dans l'article de Jean-Jacques Paul, citant une étude de JeanBernard Rasera, la nécessité d'investir en priorité dans l'enseignement primaire dans les pays les plus pauvres. En ce qui concerne l'analyse coût-bénéfice, il n'est pas nécessaire de répéter ici la liste très pertinente des questions fondamentales proposée dans son article par Robert W. Mc Meekin, que tout décideur doit se poser en la matière. On ne peut, de même, négliger la très grande complexité politique et méthodologique du développement de la formation continue des adultes, telle qu'elle est présentée par Gregory Wurzburg dans son article sur la situation dans l'Union européenne, qui pose indirectement la grande question de la faisabilité d'une réelle politique d'apprentissage à vie.

En second lieu, et indirectement lié au point précédent, les autorités responsables sont de plus en plus contraintes d'asseoir certaines de leurs décisions sur des calculs les plus exacts possible des coûts unitaires par élève et/ou par diplômé et sur l'efficacité et l'efficience même du système concerné. Le cas anglais analysé par Carol Campbell dans son article est exemplaire à cet égard: le maintien ou l'accroissement d'une partie des financements peut dépendre des résultats obtenus en ces domaines. Dans quelques pays européens, en particulier nordiques, le financement de l'enseignement supérieur tend maintenant à prendre en compte le nombre d'étudiants diplômés plutôt que le nombre d'étudiants inscrits. Aussi, l'ensemble des spécialistes partagent-ils l'opinion de François Orivel $^{1}$ : «Si l'on peut obtenir un résultat identique à un coût moindre et, si on le fait pas, on gaspille des ressources rares et on oblige la société à renoncer à d'autres utilisations 
pertinentes de ces ressources....". Depuis quelque temps déjà, les indicateurs de l'enseignement publiés par l'OCDE montrent des disparités de coût unitaire par niveau et type d'enseignement dont il n'est pas toujours aisé de découvrir la source, qui se trouve souvent dans l'histoire et la culture particulière du niveau ou type d'enseignement considéré.

9 En troisième lieu, bien que faisant clairement partie des industries de main-d'œuvre, les systèmes éducatifs ne se sont préoccupés que très tardivement de mettre sur pied de véritables politiques de gestion des ressources humaines impliquant une prise en considération et une stricte intégration d'éléments fondamentaux, tels que la formation, le recrutement, le statut et les tâches, non seulement des enseignants stricto sensu, mais également de tous les autres personnels de l'éducation, au nom d'une cohérence d'ensemble de tous les acteurs. On a traité ailleurs, en détail, de cette problématique ${ }^{2}$, dont dépend tout le fonctionnement et l'efficacité des systèmes et des politiques, et qui prenait de plus en plus de retard sur d'autres politiques de ressources de caractère sectoriel, plus aisées à conceptualiser et à mettre en œuvre, comme les ressources en capital et équipement, les structures de soutien, l'élaboration des programmes d'études, le cadre juridico-administratif, etc.

10 En quatrième lieu, ce qui vient également d'émerger et qui ne sera pas sans connaître un réajustement important de ses éléments constituants dans le cadre d'un véritable apprentissage à vie, c'est l'analyse systémique du fonctionnement des structures éducatives. Il y a, de nouveau, un lien étroit avec la production de compétences évoquée ci-dessus, mais il y a surtout dans cette approche une volonté de maîtriser les interactions entre les différents niveaux et types d'enseignement et les divers grands domaines de gestion, dont certains principes et normes de financement peuvent s'appliquer à certains niveaux et pas à d'autres et dont la complexité peut être augmentée par le degré de décentralisation du système et la persistance de cultures particulières à chaque niveau et type d'enseignement.

11 En cinquième lieu enfin et, toujours en étroite liaison avec les évolutions mentionnées cidessus et avec la tendance à une certaine décentralisation de gestion des systèmes en rapport avec une efficacité supposée plus grande de la réponse aux besoins des apprenants et de la société, s'est peu à peu développée une politique d'évaluation et de suivi. Elle se substitue progressivement aux contrôles de conformité propres aux hiérarchies bureautiques plus ou moins centralisées et reflète l'autonomie relative concédée aux établissements à la périphérie, leur responsabilité croissante et donc la nécessité de rendre des comptes à ceux qui utilisent leurs services et à ceux qui leur allouent les ressources nécessaires à leur fonctionnement. Quelle que soit l'importance des nouveaux pouvoirs attribués aux collectivités territoriales, on constate que l'établissement scolaire et/ou de formation se voit offrir des espaces d'initiative, de décision et de gestion, de plus en plus consignés dans un projet d'établissement, dont les résultats peuvent être évalués, parfois contradictoirement, à partir d'une auto-évaluation appréciée à l'aune d'un audit externe. À un niveau plus élevé, régional ou national, une synthèse régulière des résultats évalués de l'ensemble du système, sur une période donnée, peut donc être préparée, afin d'offrir aux différents responsables, acteurs et partenaires, un état du système à discuter et à éventuellement utiliser pour son amélioration.

12 Au total, comme dans le premier volet, quantitatif et qualitatif sont étroitement liés dans les études à mener, mais ce que l'on peut également conclure de ce second volet, c'est que 
la pluridisciplinarité est absolument nécessaire à la compréhension des phénomènes complexes caractérisant le fonctionnement des systèmes éducatifs. Une approche économique seule ne pourrait rendre compte des résultats que l'on recherche maintenant plus systématiquement et il apparaît que l'approche économique doit être complétée par des sciences de l'éducation et de la gestion, de la sociologie des organisations ou des sciences politiques.

\section{Contraintes et limites de l'approche économique}

Beaucoup d'articles précédents, dont ceux de Robert W. Mc Meekin et de Siegfried Hanhart, mettent l'accent sur les difficultés de mobiliser l'approche économique dans l'analyse et la mise en œuvre des politiques éducatives. On peut essayer d'en expliquer certaines raisons au travers de la culture du milieu considéré, de ses capacités d'autorégulation et de la mobilisation de moyens spécifiques.

\section{Culture fermée ou culture ouverte?}

14 Dans la mesure où le poids des enseignants, acteurs principaux des processus d'enseignement et d'apprentissage, est très important dans l'ensemble des personnels d'éducation et de formation et que, traditionnellement, cette activité de service est fortement syndicalisée et plus ou moins partie prenante aux décisions, en particulier là où le paritarisme est très développé, on peut se demander si la culture, les mentalités et les attitudes développées par ce groupe peuvent favoriser certaines évolutions mentionnées en première partie ci-dessus.

On s'interroge depuis très longtemps sur le processus de la socialisation professionnelle des enseignants et sur l'acquisition de leur identité professionnelle et, éventuellement, de celle des autres personnels de l'éducation. Malgré de nombreuses analyses, y compris comparatives, portant sur d'autres groupes professionnels, et recommandations, la formation des enseignants n'inclut pas, en général, une réelle rupture du champ d'expérience sociale et une initiation suffisamment approfondie aux problèmes de société. La sociologie des organisations, les sciences politiques, l'économie, etc. n'y ont parfois aucune place, ce qui aboutit à une perception insuffisante ou biaisée de la relation entre l'établissement d'exercice et de la société. La prégnance des disciplines et l'insuffisance de la pédagogique générale et d'une ouverture plurisdisciplinaire, laissent inachevée l'identité professionnelle acquise, la coupe des identités connexes et, en particulier, de celles de leurs collègues chargés d'administrer et de gérer le système. On recherche la "protection» disciplinaire, on ne s'intéresse éventuellement qu'aux problèmes "qualitatifs» de développement et on fait montre de frilosité, voire d'hostilité, face aux problèmes quotidiens ou plus prospectifs de gestion, laissés à ceux qui acceptent de se "salir les mains ». Les conditions de recrutement, la définition des obligations de service, le statut offert, etc. tendent à renforcer l'action individuelle, indirectement préparée par la formation et les processus d'enseignement traditionnels, au détriment d'une approche plus collégiale et ouverte des problèmes. C'est, naturellement, au moment où sont lancées des réformes offrant une autonomie relative aux établissements que l'on découvre des obstacles quasi-insurmontables dans un bref délai, comme l'absence ou l'insuffisance de culture de leadership, de culture de développement organisationnel et de culture d'évaluation. Quelle que soit la très grande 
limite actuelle de la recherche en éducation que l'on aborde ci-après, le problème évoqué ci-dessus est connu depuis longtemps et a fait l'objet, un peu partout, de propositions d'améliorations. Ces dernières s'étant rarement concrétisées, seules des études comparatives de sciences politiques sur la décision en éducation pourraient peut être nous éclairer sur la persistance de la situation. On a même parfois l'impression que le relatif isolement de l'éducateur dans son établissement semble être reflété par un isolement similaire du haut décideur politique en éducation, bien que ce dernier soit, en général, plus sensible à la rareté des ressources et à la nécessité de les gérer de la manière la plus optimale et donc de faire appel, autant que faire se peut, comme ses collègues, à l'approche économique du secteur dont il est responsable.

\section{Capacités d'auto-régulation}

16 Ce qui est apparu plus clairement depuis une quinzaine d'années - au cours desquelles la pression s'est exercée de différentes parts en faveur d'une meilleure qualité de l'enseignement délivré et d'un suivi et d'une évaluation régulière des résultats du service éducatif fourni - c'est que le système d'enseignement manquait, à de rares exceptions nationales près, de modalités propres et d'instruments adaptés à la spécificité de l'acte d'enseigner et de ses résultats. Par exemple, si les enseignants de l'éducation maîtrisaient l'évaluation traditionnelle des élèves, par contre leur faisaient défaut, là où la prégnance des examens nationaux était forte, d'autres modes d'évaluation (par exemple, la notation continue ou la validation de travaux collectifs) ou, plus généralement, leur manquait la maîtrise de l'évaluation de l'établissement, des équipes éducatives, des programmes d'études ou de tout autre domaine spécifique, indispensable à une mise en œuvre efficace des processus d'enseignement et d'apprentissage.

Dans ces circonstances, l'idée première de faire une place importante à l'auto-analyse à l'occasion de différentes modalités d'évaluation, a eu du mal à s'imposer, y compris, à la base, pour évaluer des propres pratiques. En conséquence, la réorientation marquée, au niveau européen, des inspections centrales ou générales d'un contrôle de conformité à un audit n'est pas toujours intervenue dans de bonnes conditions, faute d'un travail initial approfondi des principaux acteurs de l'établissement concerné. Or le manque d'initiative locale en ce domaine a pour conséquence quasi-automatique que seule l'évaluation externe a prévalu, avec tous les risques impliqués par l'absence de point de vue des principaux acteurs et de dialogue. En outre, l'insuffisante multi-dimensionnalité de l'évaluation peut conduire les divers partenaires à s'engager dans des éventuelles réorientations manquant de bases solides. Ce qui a été beaucoup plus grave - et le reste encore actuellement - c'est que la faiblesse traditionnelle de la recherche-développement (R\&D) dans et sur les systèmes éducatifs n'a pas toujours permis de construire, après analyse et réflexion, des instruments et des stratégies d'évaluation reflétant les finalités des systèmes et la spécificité des processus d'enseignement et d'apprentissage mis en œuvre. En leur absence, la seule ressource des systèmes, confrontés à la volonté politique de faire état des résultats obtenus, a été d'importer des méthodes et des stratégies employées dans d'autres secteurs d'activité (en particulier les entreprises) qui n'étaient parfois pas adaptées au monde éducatif. Sans vouloir en inférer que des théories générales d'administration et de gestion ne s'appliqueraient pas à ce secteur, on peut néanmoins avancer que si les dépenses de R\&D en éducation se situaient entre 3 et $5 \%$ des dépenses courantes, comme beaucoup de spécialistes le souhaitent, les systèmes 
éducatifs auraient certainement à leur disposition beaucoup plus de moyens de s'autoréguler.

18 Mais encore conviendrait-il, lorsque des moyens internes au système existent, qu'ils soient concrètement mobilisés ou développés. L'exemple français récent est instructif à cet égard. En ce qui concerne l'enseignement supérieur, une réforme récente des structures du ministère a supprimé l'Observatoire des coûts, organisme peu répandu ailleurs, qui avait le mérite, sur une base de volontariat, d'analyser en détail les coûts unitaires des étudiants ou diplômés selon les filières d'études et qui pouvait compléter utilement les évaluations institutionnelles du Comité national d'évaluation. Lors d'un séminaire à l'Institut des entreprises de l'université de Poitiers en 1997, consacré à l'évaluation des universités, des responsables de la préparation des contrats quadriennaux entre le ministère et les universités avaient avoué que le manque de temps ne leur permettait pas de mobiliser les rapports d'évaluation d'origine très variable pouvant intéresser les universités concernées. Enfin, toujours en France, à l'occasion de la réforme mentionnée ci-dessus, la transformation de la direction de l'Évaluation et de la Prospective en une direction de la Programmation et du Développement a eu pour conséquence de freiner la dynamique de l'évaluation initiée, depuis plusieurs années, par la direction précédente. Il est donc très intéressant de noter, à cette occasion, que cette direction qui passait à l'époque pour un modèle dans un certain nombre de pays de l'OCDE, a vu son influence décliner, prouvant en cela qu'il n'est jamais certain qu'une innovation politique jugée par beaucoup opportune à l'époque de sa création et de son développement puisse se maintenir.

En matière de ressources financières, on peut juger des capacités d'autorégulation au travers d'une nouvelle problématique, celle de la recherche de "gisements d'économies ", comme source alternative de moyens financiers additionnels, en l'absence de possibilité d'accroissement de ressources externes. Dans la quasi-totalité des cas, ceci signifie un accroissement de productivité du système en termes de respect des durées d'études, d'augmentation optimale des élèves par filières, de regroupement de filières et d'établissements, d'investissement favorisant les économies d'échelle systématiques, etc. Très souvent la segmentation des grandes filières d'études (en particulier dans l'enseignement supérieur), les intérêts acquis par certains établissements (éventuellement de prestige et parfois de petite taille), le refus d'une réaffectation géographique de la part des personnels, etc. empêchent de dégager les sommes nécessaires pour le développement du système. Ceci a alors pour conséquence l'accroissement des inégalités sous deux formes. D'une part, des établissements bénéficiant de réelles "rentes de situation " se permettent des dépenses par élève beaucoup plus élevées qu'ailleurs. D'autre part, pour compenser ce manque à gagner en l'absence de mesures adéquates, on tend soit à appauvrir certains autres établissements soit on s'engage subrepticement ou formellement dans une politique d'appel à des contributions des élèves et/ou de leur famille, sans toujours être attentif aux moyens dont ils disposent.

20 Enfin, comme le montre bien l'article de Robert W. Mc Meekin, encore faut-il que le décideur politique soit convaincu qu'il lui est utile de s'entourer d'une équipe de spécialistes qui, grâce en particulier à une approche économique, l'aidera à formuler, mettre en œuvre et suivre une innovation majeure dans le système. L'exemple donné concernant l'Amérique latine peut être aisément étendu au reste du monde. Certes, il y a dans certaines régions des pénuries de spécialistes de la dimension économique de 
l'éducation, mais là ou un groupe de spécialistes existe, il y a beaucoup plus de chances de le trouver ailleurs que dans les sphères ministérielles, à titre de conseiller.

\section{Moyens disponibles}

21 L'insuffisance de la $R \& D$ évoquée ci-dessus, conjuguée à un manque de ressources humaines et matérielles, si ce n'est à une volonté idéologique, a naturellement des conséquences sur la disponibilité d'instruments ou organismes à même de soutenir l'approche économique du fonctionnement de l'enseignement. Depuis 1990, par exemple, les pays d'Europe centrale et orientale, échaudés par le système antérieur liant éducation et emploi, ne se sont pas réellement dotés d'organismes de type «Centre d'études et de recherches sur les qualifications " pour aider les acteurs en ce domaine à mieux orienter leur offre. Dans ce même groupe de pays, les nouvelles législations sur l'enseignement supérieur se sont contentées d'importer le matériel américain d'accréditation des institutions, sans mettre sur pied un système réel d'évaluation. Il y a quelques années, l'autorisation donnée en République tchèque à des établissements privés subventionnés de contribuer à l'enseignement scolaire, sans aucun cadre réglementaire de suivi et de contrôle, a abouti à une prolifération d'établissements de valeur et de taille inégale, émargeant au budget public, etc. Encore actuellement, beaucoup de pays dans le monde et, pas seulement les plus pauvres, manquent d'unités centrales ou régionales bien équipées pour traiter statistiques et indicateurs de performance sur des bases de plus en plus comparatives, pour répondre aux nouveaux besoins d'intégration régionale. Comme on l'a montré ailleurs ${ }^{3}$, la mise en place d'un système d'évaluation suppose un minimum de moyens qui ne sont pas toujours réunis. Au-delà même de l'infrastructure, les débats continuent sur la signification et les normes d'utilisation des divers types d'indicateurs mis à la disposition des divers acteurs et partenaires ${ }^{4}$. Enfin, les rapports sur l'état de l'enseignement et de la formation, supposés mettre à la disposition de tous les intéressés, à espaces réguliers, une analyse approfondie des systèmes et des suggestions d'améliorations, ont du mal à voir le jour sur le modèle, par exemple, de ce qui se faisait il y a quelques années en Californie ${ }^{5}$. En premier lieu, les pays à système fragmenté ont du mal à regrouper les informations correspondantes. En second lieu, tout ce qui concerne les données sur l'éducation des adultes est encore dans l'enfance, compte tenu de la large décentralisation de l'offre, et rend encore plus lointaine l'obtention de véritables bilans de l'apprentissage à vie pour tous. En troisième lieu, la très grande variété des contextes où des institutions pourraient répondre au besoin évoqué ci-dessus soulève quatre problèmes : celui du degré d'autonomie dont devrait bénéficier l'institution responsable, celui de la composition, la plus représentative possible, de son conseil d'administration; celui des moyens devant lui être attribués; celui enfin du suivi scientifique de son activité.

22 Au total, les quelques exemples ci-dessus témoignent de la très grande complexité de la mise en place d'une stratégie d'utilisation plus systématique qu'actuellement de l'approche économique dans le développement des politiques éducatives.

La difficulté d'intégration de la dimension économique dans l'analyse et le développement des politiques éducatives a pour première origine une conception uniquement qualitative des processus d'enseignement et d'apprentissage, quel que soit leur type ou leur niveau. Ce point de vue est très largement partagé par les principaux acteurs des systèmes éducatifs, y compris, bien souvent, par les décideurs politico- 
administratifs et les administrateurs-gestionnaires, d'autant plus sensibles à cette opinion très largement majoritaire qu'ils sont parfois eux-mêmes issus du corps enseignant. Il est très difficile de dire à l'heure actuelle si, dans l'avenir plus ou moins lointain, les nouveaux moyens de communication et d'information ainsi que des changements dans les relations d'apprentissage, pouvant être induits par la variation de la taille des groupes d'apprenants, modifieraient - au moins pour certains niveaux et types d'enseignement et de formation - le caractère d'« industrie de main-d'œuvre » des systèmes éducatifs, actuellement accepté, voire intériorisé - sauf rares exceptions -, par l'ensemble des parties prenantes. Dans la meilleure des hypothèses, l'émergence de l'apprentissage à vie, comme cadre de développement des politiques, devrait donner une nouvelle impulsion à la recherche-développement et à des stratégies d'innovations s'appuyant, en particulier, sur l'approche économique du fonctionnement des systèmes, intégrée à une approche pluridisciplinaire. Dans le cas contraire, on pourrait faire son deuil de la cohérence et de l'intégration de systèmes et de leur capacité à gérer les rapports de plus en plus complexes entre qualité, équité et efficacité. Les risques de fragmentation, voire d'éclatement des systèmes, déjà à l'œuvre dans certains endroits, augmenteront et les efforts divers d'analyse systémique, d'évaluation, de calculs coûtbénéfice, etc. subsistants, ne feraient que légitimer une évolution néfaste pour le développement, le plus harmonieux possible, tant des individus que de la société.

\section{NOTES}

1. François Orivel, «L'allocation des ressources par élève dans le système éducatif français. Point de vue d'un économiste ", Revue française de pédagogie, n 105, octobre-décembre 1993.

2. Pierre Laderrière, «Une problématique nouvelle : la gestion des ressources humaines dans l'enseignement ", Recherche et Formation, n 30, 1999.

3. Pierre Laderrière, «Évaluer, comment? ", in « Éduquer en Europe », L'événement européen Initiatives et débats, Le Seuil, décembre 1990.

4. Benoît Bayenet, Olivier Débande, Françoise Thys-Clément, «Évaluation des systèmes éducatifs : des méfaits à l'utilité d'indicateurs de comparaison ", in: Éducation et Sociétés, $\mathrm{n}^{\circ} 1$, 1998.

5. James W. Guthrie, «L'évolution des politiques économiques et son incidence sur l'évaluation des systèmes éducatifs ", in Évaluer et réformer les systèmes éducatifs, Paris, OCDE, 1996

\section{RÉSUMÉS}

Prenant pour point de départ le contenu du dossier, l'article traite de l'enracinement de la dimension économique en insistant sur ses deux volets : la "production » de compétences et la 
combinaison optimale des ressources, puis examine les obstacles rencontrés en termes de culture dominante, de capacités d'autorégulation et de moyens disponibles.

Using the contents of this study as a starting point, the article deals with the deep-rooted nature of the economic dimension in its two parts: the " production » of skills and the best combination of resources. Then it examines the obstacles encountered in terms of the dominant culture, autoregulatory capacities and available means.

Partiendo del contenido del dossier, el artículo trata del arraigo de la cuestión económica insistiendo en dos aspectos: la « producción » de las competencias y la combinación óptima de los recursos. A continuación examina los obstáculos que suelen encontrarse en cuestión de cultura dominante, de capacidades de autoregulación y de medios disponibles.

INDEX

Mots-clés : compétence, formation continue, formation professionnelle, relation formationemploi, économie

\section{AUTEUR}

PIERRE LADERRIÈRE

Consultant en politiques d'enseignement. 